\title{
Acyclic retinoid inhibits angiogenesis by suppressing the MAPK pathway
}

\author{
Yusuke Komi ${ }^{1,2}$, Yukihisa Sogabe ${ }^{1}$, Naoto Ishibashi ${ }^{3}$, Yasufumi Sato ${ }^{4}$, Hisataka Moriwaki ${ }^{5}$, Kentaro Shimokado ${ }^{2}$ \\ and Soichi Kojima'
}

Acyclic retinoid (ACR) is currently under clinical trial as an agent to suppress the recurrence of hepatocellular carcinoma (HCC) through its ability to induce apoptosis in premature HCC cells. ACR has an anticancer effect in vivo as well, although it shows weak apoptosis-inducing activity against mature HCC cells, suggesting the existence of an additional action mechanism. In this study, we investigated the antiangiogenic activity of ACR. ACR inhibited angiogenesis within chicken chorioallantoic membrane (CAM) in as similar a manner as all-trans retinoic acid (atRA). Although suppression of angiogenesis by atRA was partially rescued by the simultaneous addition of angiopoietin-1, suppression of angiogenesis by ACR was not rescued under the same condition at all. Conversely, although suppression of angiogenesis by ACR was partially inverted by the simultaneous addition of vascular endothelial growth factor (VEGF), suppression of angiogenesis by atRA was not affected under the same condition. These results suggested that mechanisms underlying the suppression of angiogenesis by ACR and atRA were different. ACR selectively inhibited the phosphorylation of VEGF receptor 2 (VEGFR2) and of extracellular signal-regulated kinase (ERK) without changing their protein expression levels, and inhibited endothelial cell growth, migration, and tube formation. The inhibition of the phosphorylation of ERK, endothelial growth, migration, tube formation, and angiogenesis by ACR was rescued by the overexpression of constitutively active mitogen-activated protein kinase (MAPK). Finally, ACR, but not atRA, inhibited HCC-induced angiogenesis in a xenografted CAM model. These results delineate the novel activity of ACR as an antiangiogenic through a strong inhibition of the VEGFR2 MAPK pathway.

Laboratory Investigation (2010) 90, 52-60; doi:10.1038/labinvest.2009.110; published online 19 October 2009

KEYWORDS: ACR; HCC; MAPK pathway; phosphorylation; tumor angiogenesis; VEGF receptor

Angiogenesis has an important role in tumor growth by supplying nutrients and providing a route for metastasis. ${ }^{1}$ Therefore, tumor angiogenesis is a good target for the treatment of solid cancers. Tumor cells induce angiogenesis by producing and releasing several angiogenic factors, such as vascular endothelial growth factor (VEGF), basic fibroblast growth factor (bFGF), and angiopoietins (Angs). ${ }^{1}$ The VEGF/ VEGF receptor (VEGFR) signaling pathway is essential for drawing endothelial cells from preexisting blood vessels and in stimulating their growth, ${ }^{2}$ whereas the Ang/Tie2 signaling pathway is important for sustaining the interaction between endothelial and mural cells and stabilizing the vasculature.
Retinoids (vitamin A and its derivatives) are natural fatsoluble hormones, the biological effects of which are believed to be mediated, all or in part, by the modulation of target gene expression through two families of nuclear receptors: retinoic acid receptors (RARs) and retinoid $\mathrm{X}$ receptors (RXRs). ${ }^{3}$ Retinoids exert antitumor activity by modifying the transactivation of $\mathrm{p} 21^{\mathrm{CIP} 1}$, interferon receptor, and signal transduction and activator of transcription. ${ }^{4,5}$ We previously reported that all-trans retinoic acid (atRA) inhibits angiogenesis on chorioallantoic membrane (CAM) through disruption of vascular remodeling by inducing Ang2 expression and suppressing Ang/Tie2 signaling. ${ }^{6}$ Acyclic retinoid (ACR)

\footnotetext{
${ }^{1}$ Molecular Ligand Biology Research Team, Chemical Genomics Research Group, Chemical Biology Department, RIKEN Advanced Science Institute, Saitama, Japan; ${ }^{2}$ Department of Vascular Medicine and Geriatrics, Tokyo Medical and Dental University Graduate School, Tokyo, Japan; ${ }^{3}$ Tokyo New Drug Research Laboratory, Pharmaceutical Division, Kowa, Tokyo, Japan; ${ }^{4}$ Department of Vascular Biology, Institute of Development, Aging and Cancer, Tohoku University, Sendai, Japan and ${ }^{5}$ Department of Gastroenterology, Gifu University School of Medicine, Gifu, Japan

Correspondence: Professor S Kojima, PhD, Molecular Ligand Biology Research Team, Chemical Genomics Research Group, Chemical Biology Department, RIKEN Advanced Science Institute, 2-1 Wako, Saitama 351-0198, Japan.

E-mail: skojima@postman.riken.go.jp

Received 3 February 2009; revised 20 July 2009; accepted 31 July 2009
} 
is a synthetic retinoid and activates the RAR and RXR. ${ }^{7}$ Oral administration of ACR for 12 months significantly reduced the incidence of post-therapeutic recurrence of hepatocellular carcinoma (HCC) compared with the placebo group. ${ }^{8}$ In this study, ACR did not cause the typical toxic effects observed with conventional retinoids. ${ }^{8}$ Now, ACR is under clinical trials as a chemopreventive drug against the recurrence of HCC. Nuclear receptor RXR in HCC is highly phosphorylated through the Ras-extracellular signal-regulated kinase (ERK) pathway, inactivated, and accumulates in the line as a dominant-negative receptor. ${ }^{9,10}$ ACR inhibits the phosphorylation of RXR by inactivating the Ras-ERK pathway, recovering transactivation by retinoic acid, and induces apoptosis in human HCC cell lines. ${ }^{9,10}$ ACR also has an anticancer effect in vivo. ${ }^{11}$ However, it exerts only weak apoptosis-inducing activity against mature HCC cells in vivo. This result suggests an existence of an additional molecular mechanism underlying the anticancer effect of ACR. Therefore, we predicted that ACR might have antiangiogenic activity.

Herein, we found that in contrast to the antiangiogenic mechanism of atRA, ACR inhibited angiogenesis through the inhibition of the VEGF receptor mitogen-activated protein kinase (MAPK) pathway. Moreover, ACR suppressed HCCinduced angiogenesis in a xenografted CAM model. These results suggest that ACR will also be clinically useful as an antiangiogenic agent, in addition to its current usage as a chemopreventive agent.

\section{MATERIALS AND METHODS Reagents}

Acyclic retinoid (2E,4E,6E,10E)-3,7,11,15-tetramethylhexadeca2,4,6,10,14-pentaenoic acid) was provided by Kowa (Tokyo, Japan). AtRA was purchased from Sigma-Aldrich (St Louis, MO, USA). ACR was dissolved in ethanol and dimethyl sulfoxide (DMSO) to yield stock solutions of $10 \mathrm{mM}$ and $1 \mathrm{M}$, respectively, whereas atRA was dissolved in ethanol to yield a stock solution of $17 \mathrm{mM}$.

\section{Chicken CAM Assay}

In vivo antiangiogenic activity of ACR and atRA was assessed by CAM assay as described previously. ${ }^{12}$ In brief, fertilized Dekalb chicken eggs (Omiya Kakin, Saitama, Japan) were placed in a humidified egg incubator. After a 4.5-day incubation at $38^{\circ} \mathrm{C}$, a $1 \%$ solution of methylcellulose containing ACR or atRA at various concentrations was loaded inside a silicon ring that was placed onto the surface of CAM. After a further incubation for 2 days, a fat emulsion was injected into the chorioallantois, so that the vascular networks stood out against the white background of the lipid. Antiangiogenic responses were evaluated under a stereomicroscope and photographed with a $\times 7.25$ objective. Quantitative analyses were carried out with angiogenesismeasuring software (ver.2.0; KURABO, Osaka, Japan). ${ }^{12}$

\section{Matrigel Plug Assay}

Matrigel (BD Biosciences, Bedford, MA, USA) was mixed with 200 units/ml heparin (Nacalai Tesque, Kyoto, Japan), with and without $50 \mathrm{ng} / \mathrm{ml}$ VEGF (Pepro Tech, Rocky Hill, NJ, USA) and $5 \mu \mathrm{M}$ ACR in $0.1 \%$ DMSO. The matrigel mixture was injected subcutaneously into 5 -week-old female C57BL/6 mice (Charles River, Yokohama, Japan). The mice were killed 7 days later. The matrigel plugs were removed and fixed in $4 \%$ paraformaldehyde for $4 \mathrm{~h}$, dehydrated through a graded ethanol series, and embedded in paraffin (Nacalai Tesque). Vertical sections ( $5 \mu \mathrm{m}$ ) were mounted on slides and stained with hematoxylin and eosin, and observed under an inverted microscope (model DM IRB, Leica Microsystems, Wetzlar, Germany).

\section{Cell Cultures}

Human umbilical vein endothelial cells (HUVECs) and bovine aortic endothelial cells were cultured as described. ${ }^{12}$ HepG2 cells, human HCC, were cultured in Dulbecco's modified Eagle's medium (Sigma-Aldrich) supplemented with $10 \%$ fetal calf serum.

\section{Transfection and Luciferase Assay}

Transfection into HUVECs was carried out using a combination of LipofectAMINE 2000 Plus reagent (Invitrogen) and a constitutively active MAPK kinase vector $(1.5 \mu \mathrm{g}$ each per $35-\mathrm{mm}$ dish). ${ }^{13}$

\section{Western Blotting Analysis}

After rinsing several times with TBS $(20 \mathrm{mM}$ Tris- $\mathrm{HCl}$, $137 \mathrm{mM} \mathrm{NaCl}$ ), cells were lysed in $1 \%$ Triton X-100 in $20 \mathrm{mM}$ HEPES, $\mathrm{pH}$ 6.8, containing Complete protease inhibitor cocktail (1 tablet per $50 \mathrm{ml}$; Roche, Indianapolis, IN, USA), $1 \mathrm{mM}$ EDTA, $1 \mathrm{mM}$ PMSF, and $0.5 \mathrm{mM} \mathrm{Na}_{3} \mathrm{VO}_{5}$, and directly subjected to western analysis using phospho-VEGFR2-specific antibodies (1:1000 dilution; Cell Signaling Technology, Danvers, MA, USA), phospho-FGFR1-specific antibodies (1:1000 dilution; Cell Signaling Technology), or phosphoERK-specific antibodies (1:2000 dilution, Cell Signaling Technology). Cell lysates were also subjected to western analysis using antibodies to VEGFR2, FGFR1, and ERK. Immunoreactive bands of proteins were detected with ECL-Plus chemiluminescence reagents (GE Healthcare, Buckinghamshire, UK).

\section{In Vitro Tube Formation Assay}

Tube formation by HUVECs on matrigel was assessed as described previously. ${ }^{14}$ Unpolymerized matrigel (Becton Dickinson, Bedford, MA, USA) was diluted to a final concentration of $5 \mathrm{mg} / \mathrm{ml}$ with MCDB-131 medium, aliquoted $150 \mu \mathrm{l}$ each into 24 -well plates, and allowed to polymerize for $30 \mathrm{~min}$ at $37^{\circ} \mathrm{C}$. HUVECs were transfected with a constitutively active MAPK kinase-expressing vector. Two days later, HUVECs were seeded onto the polymerized gel at $2 \times 10^{5}$ cells/well; thereafter, $100 \mathrm{ng} / \mathrm{ml}$ VEGF, $1 \mu \mathrm{M}, 5 \mu \mathrm{M}$, 
and $10 \mu \mathrm{M}$ ACR and/or atRA were added, and incubated for $6 \mathrm{~h}$. In vitro tube formation was examined under a phase-contrast microscope and photographed with a $\times 10$ objective.

\section{HCC-Induced Angiogenesis in a Xenografted CAM Model}

Hepatocellular carcinoma-induced angiogenesis in a xenografted CAM model was assessed as previously described. ${ }^{15,16}$ HepG2 cell suspensions with or without $5 \mu \mathrm{M}$ ACR or atRA were delivered at $4 \times 10^{5}$ cells per embryo onto the top of the CAM on day 8 using a gelatin sponge, called Gelform (Pfizer, New York, NY, USA) implant. After a further 4-day incubation, a fat emulsion was injected into the chorioallantois, so that the vascular networks stood out against the white background of the lipid. Antiangiogenic responses were evaluated under a stereomicroscope and photographed with a $\times 25$.

\section{Statistical Analysis}

Data are expressed as means \pm s.d. Statistical significance was assessed by one-way analysis of variance, followed by Shaffer's $t$-test.

\section{RESULTS}

\section{Comparison Between the Effects of ACR and atRA on Blood Vessel Formation in CAM}

To determine whether ACR could inhibit in vivo angiogenesis, we carried out CAM assay (Figure 1). The formation of intricate vascular networks, developing within control CAM

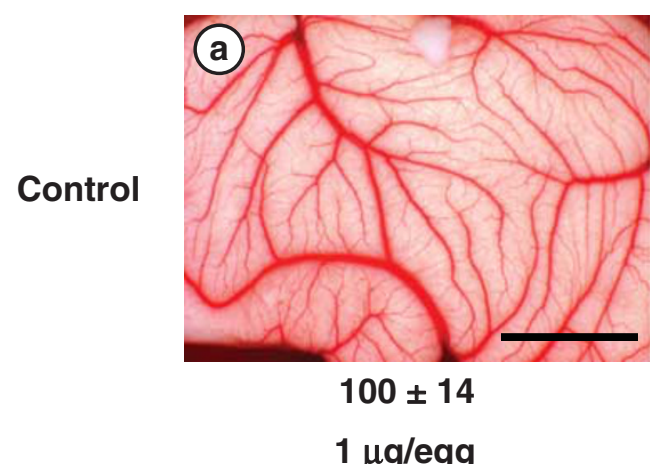

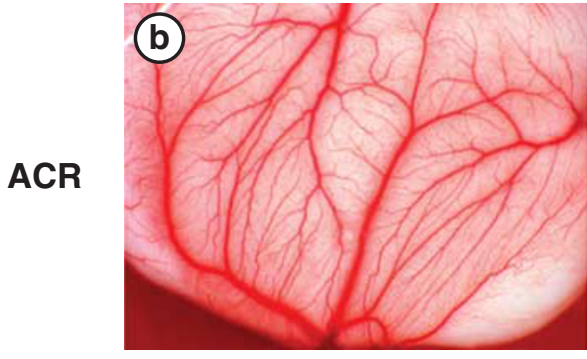

$102 \pm 15$

$100 \mathrm{ng} / \mathrm{egg}$

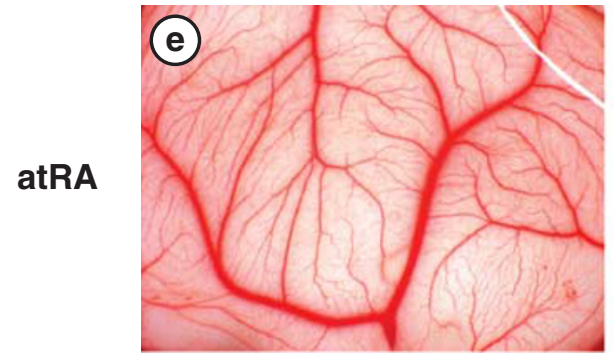

$96 \pm 14$
$5 \mu \mathrm{g} / \mathrm{egg}$

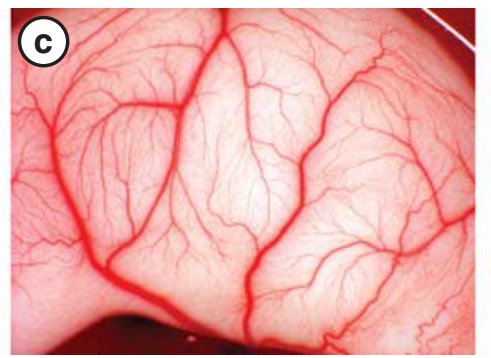

$53 \pm 8^{*}$

$500 \mathrm{ng} / \mathrm{egg}$

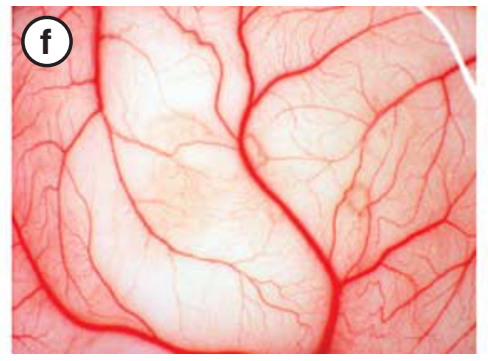

$50 \pm 5^{*}$
$10 \mu \mathrm{g} / \mathrm{egg}$
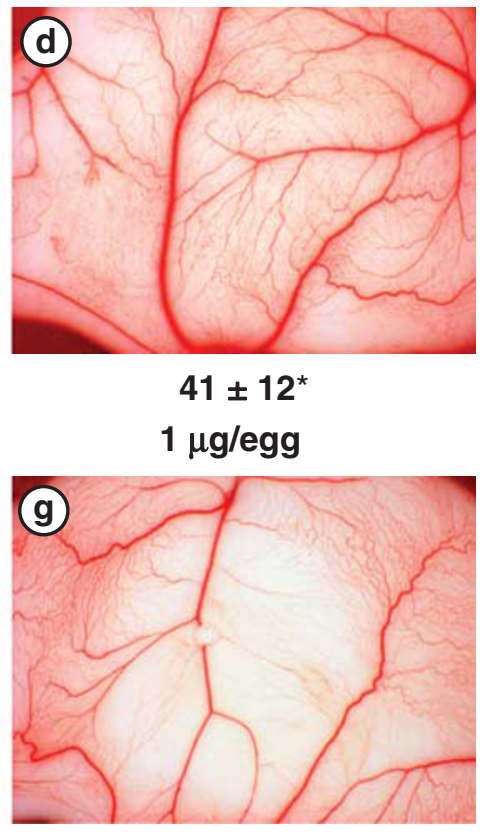

$36 \pm 10^{*}$

Figure 1 Suppression of in vivo angiogenesis in CAM by ACR and atRA. The 4.5-day-old CAMs were treated with ACR and atRA for $48 \mathrm{~h}$, and then patterns of angiogenesis were photographed. Panel (a), vehicle (1\% ethanol plus 1\% DMSO); panel (b), $1 \mu \mathrm{g} / \mathrm{egg}$ ACR; panel (c), $5 \mu \mathrm{g} / \mathrm{egg}$ ACR; panel (d), $10 \mu \mathrm{g} / \mathrm{egg} \mathrm{ACR}$; panel (e), $100 \mathrm{ng} / \mathrm{egg}$ atRA; panel (f), $500 \mathrm{ng} / \mathrm{egg}$ atRA; panel (g), $1 \mu \mathrm{g} / \mathrm{egg}$ atRA. Scale bar, $5 \mathrm{~mm}$. Total numbers of branches of blood vessels were analyzed with angiogenesis-measuring software and are shown under each panel. A total of 12 eggs ( 6 eggs per experiment $\times 2$ experiments) were evaluated and representative results are shown. An asterisk indicates a significant difference $(P<0.05)$ from the control. 
A

$(-)$

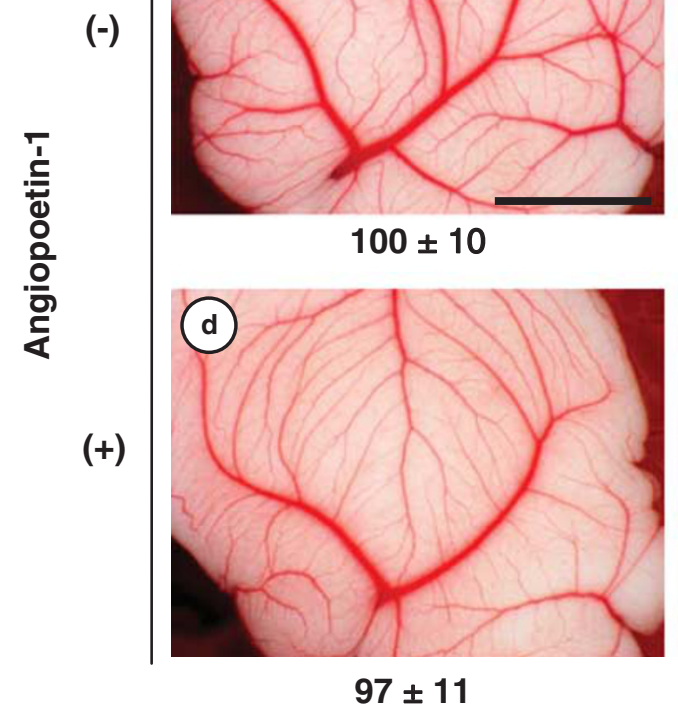

control

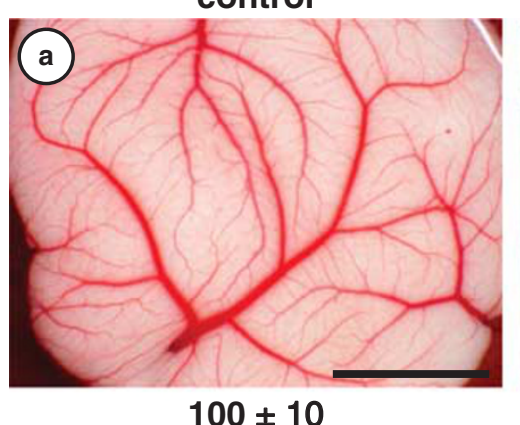

$97 \pm 11$

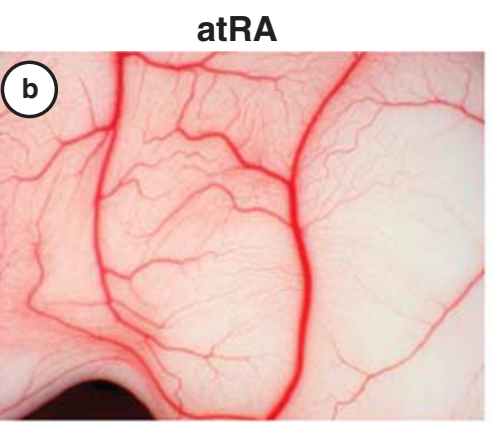

$54 \pm 19^{*}$

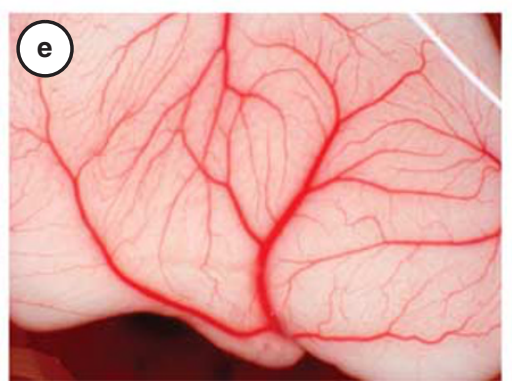

$73 \pm 8^{\star}$
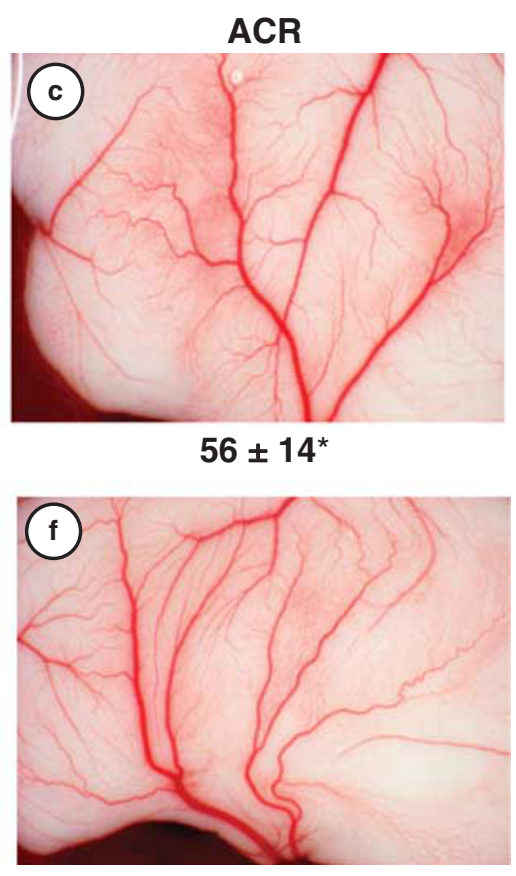

$59 \pm 20^{*}$

Figure 2 The antiangiogenic effect of atRA, but not ACR, was rescued by simultaneous treatment with Ang1 in CAM. (A) The 4.5-day-old CAMs were treated with ACR and atRA for $48 \mathrm{~h}$ and then patterns of angiogenesis were photographed. Panel (a), vehicle (1\% ethanol plus 1\% DMSO); panel (b), 500 ng/egg atRA; panel (c), $3 \mu \mathrm{g} / \mathrm{egg}$ ACR; panel (d), vehicle plus $300 \mathrm{ng} / \mathrm{egg}$ human recombinant Ang1; panel (e), $500 \mathrm{ng} / \mathrm{egg}$ atRA plus $300 \mathrm{ng} / \mathrm{egg}$ human recombinant Ang1; panel (f), $3 \mu \mathrm{g} / \mathrm{egg}$ ACR plus $300 \mathrm{ng} / \mathrm{egg}$ human recombinant Ang1. Scale bar, $5 \mathrm{~mm}$. Total numbers of branches of blood vessels were analyzed with angiogenesis-measuring software and are shown under each panel. A total of 18 eggs ( 6 eggs per experiment $\times 3$ experiments) were evaluated and representative results are shown. An asterisk indicates a significant difference $(P<0.05)$ from the control. This result shows the representative result from three independent experiments, all of which gave similar results.

(Figure 1, panel a), was suppressed with ACR in a dosedependent manner at concentrations of $1-10 \mu \mathrm{g} / \mathrm{egg}$ (0.3-3.3 mM inside the ring) (Figure 1, panels b-d) and with atRA in a dose-dependent manner at about 10 times lower concentrations of $100-1000 \mathrm{ng} / \mathrm{egg}(33-333 \mu \mathrm{M})$ (Figure 1, panels $\mathrm{e}-\mathrm{g}$ ). Although the inhibition of angiogenesis with atRA was partially rescued by simultaneous treatment with Ang1 at a concentration of $300 \mathrm{ng} / \mathrm{egg}$ as consistently as we reported previously ${ }^{6}$ (Figure $2 \mathrm{~A}$, panel e), inhibition of angiogenesis with ACR was not rescued with Ang1 at all (Figure 2A, panel f). Furthermore, although atRA stimulated the transactivation activity of the Ang 2 promoter twofold (Supplementary Figure 1, column 2), ACR hardly showed such an activity (Supplementary Figure 1, columns 3 and 4). On the other hand, inhibition of angiogenesis with ACR, but not with atRA, was rescued by simultaneous treatment with VEGF (compare Figure 3A, panels e and $\mathrm{f}$ ). To determine whether ACR might inhibit VEGF-induced blood vessel formation in vivo, we examined the effect of ACR in the matrigel plug assay (Figure 3B). Invasion of cells into gels was observed in the control matrigel that contained VEGF without ACR (panel a). When ACR was included in the matrigel at a concentration of $5 \mu \mathrm{M}$, the VEGF-induced invasion of cells was inhibited by about 54\% (panel b).

\section{Effect of ACR and atRA on Endothelial Cell Growth, Migration, and Tube Formation}

We investigated the molecular mechanism by which ACR inhibited angiogenesis. First, we compared the effect of ACR and atRA on vascular endothelial cells. ACR $(5 \mu \mathrm{M})$ suppressed the growth, migration, and tube formation (Figure 4A, lane 2, closed column; Figure 4B, lane 2, closed column; Figure $4 \mathrm{C}$, panel b, respectively). These suppressive effects by ACR were, all or in part, rescued by overexpressing a constitutive active MEK gene (Figure 4A, lane 2, open column; Figure 4B, lane 2, open column; Figure 4C, panel e, respectively). Conversely, atRA did not suppress, rather it enhanced all of them (Figure 4A, lane 3, closed column; Figure 4B, lane 3, closed column; Figure 4C, panel c, respectively).

\section{ACR Suppressed Phosphorylation of VEGFR2 and ERK}

Next, we examined the effect of ACR and atRA on the phosphorylation of angiogenic growth factor receptors expressed by endothelial cells. As seen in the upper panel of Figure $5 \mathrm{a}$, induction of phosphorylated $230 \mathrm{kD}$ VEGFR2 after VEGF treatment was blocked to about $20 \%$ by pretreatment with $5 \mu \mathrm{M}$ ACR for $24 \mathrm{~h}$ (compare lanes 4 with 5). In contrast, pretreatment with $5 \mu \mathrm{M}$ atRA for $24 \mathrm{~h}$ did not block the 
A

$(-)$

$(+)$
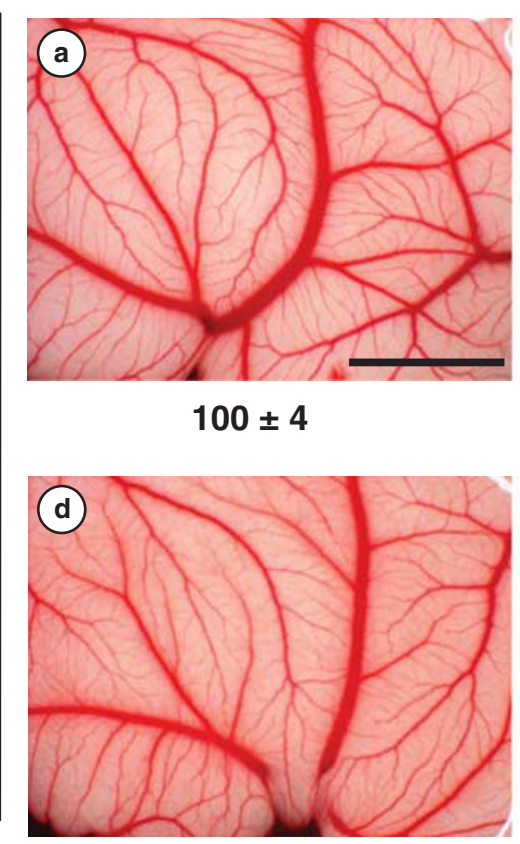

$101 \pm 18$
ACR

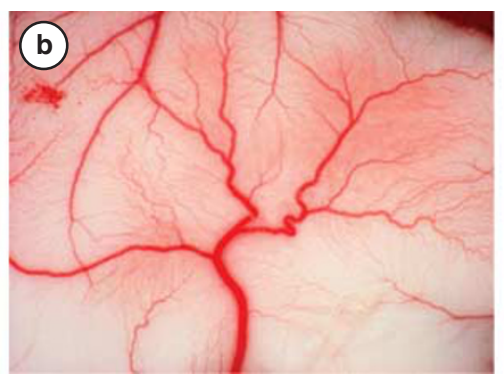

$42 \pm 13^{*}$

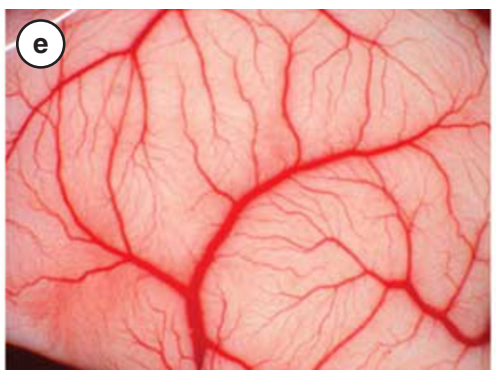

$71 \pm 16^{*}$
atRA

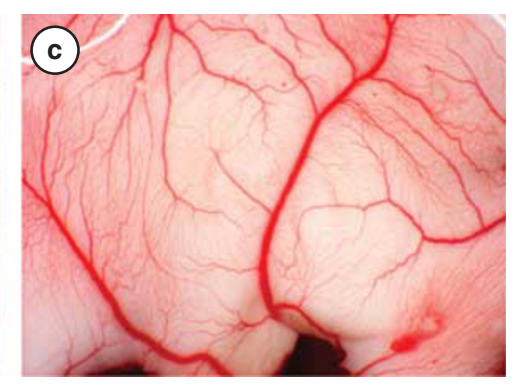

$33 \pm 14^{*}$

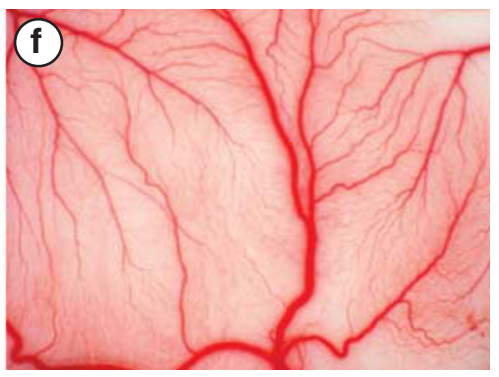

$37 \pm 15^{*}$

B

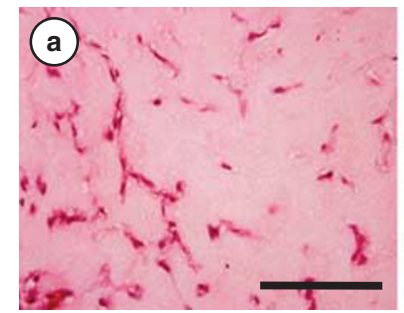

$101 \pm 15$

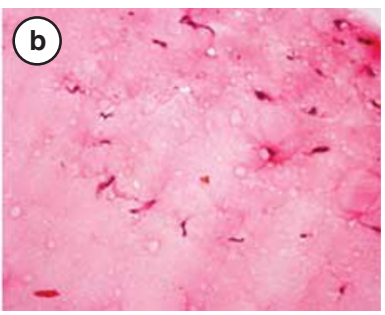

$46 \pm 25^{*}$

Figure 3 The antiangiogenic effect of ACR, but not of atRA, was rescued by simultaneous treatment with VEGF in CAM (A). The 4.5-day-old CAMs were treated with ACR and atRA for $48 \mathrm{~h}$ and then patterns of angiogenesis were photographed. Panel (a), vehicle (1\% ethanol plus $1 \%$ DMSO); panel (b), $3 \mu \mathrm{g} / \mathrm{egg}$ ACR; panel (c), $500 \mathrm{ng} / \mathrm{egg}$ atRA; panel (d), vehicle plus $1 \mathrm{ng} / \mathrm{egg}$ mouse recombinant VEGF; panel (e), $3 \mu \mathrm{g} / \mathrm{egg}$ ACR plus $1 \mathrm{ng} / \mathrm{egg}$ mouse recombinant VEGF; panel (f) $500 \mathrm{ng} / \mathrm{egg}$ atRA plus $1 \mathrm{ng} / \mathrm{egg}$ mouse recombinant VEGF. Scale bar, $5 \mathrm{~mm}$. Total numbers of branches of blood vessels were analyzed with angiogenesis-measuring software and are shown under each panel. A total of 18 eggs (6 eggs per experiment $\times 3$ experiments) were evaluated and representative results are shown. (B) Matrigel plug assay: matrigel plugs containing $50 \mathrm{ng} / \mathrm{ml}$ VEGF $\pm 5 \mu \mathrm{M}$ ACR were implanted into mice subcutaneously. One week later, matrigel plugs were collected and stained with hematoxylin and eosin (panels a and b). Panel a, VEGF alone (control); panel $\mathbf{b}$, VEGF plus ACR. Representative data from a total of nine micrographs ( 3 fields $\times 3$ mice) are presented. Scale bar, $500 \mu \mathrm{m}$. The number of invading cells in each micrograph was counted and the relative values are presented as percentages under each photograph. An asterisk indicates a significant difference $(P<0.05)$ from the control. Panels $\mathbf{A}$ and $\mathbf{B}$ show representative results from two independent experiments, both of which gave similar results.

phosphorylation of VEGFR2 but rather increased it (compare lanes 4 with 6). Both ACR and atRA decreased the expression of VEGFR2 to about 70 and $60 \%$, respectively, without VEGF treatment (compare lanes 1 with 2 and 3). However, this effect was not obvious in cells treated with VEGF (compare lanes 4-6). ACR did not affect the binding of VEGF to VEGFR2, nor did it affect VEGF mRNA levels (data not shown). On the other hand, pretreatment with ACR or atRA did not block the phosphorylation of FGFR1 but rather enhanced it (Figure 5b). Whereas ACR inhibited the phosphorylation of Ras, it did not inhibit the phosphorylation of Akt (Supplementary Figure 2). In addition, pretreatment with $5 \mu \mathrm{M}$ ACR, but not with atRA, significantly inhibited the phosphorylation of ERK, which is induced downstream of VEGF stimuli (Figure 6a, lanes 5 and 6 in upper panel, respectively). The inhibition by ACR was inverted by the overexpression of constitutively active MAPK kinase in HUVECs (Figure 6b, lane 4 in upper panel).

\section{Effect of ACR and atRA on HCC-Induced Angiogenesis in a Xenografted CAM Model}

To confirm whether ACR and atRA have anti-HCC-induced angiogenic activity in vivo, we investigated the effect of ACR and atRA on HCC-induced angiogenesis in a xenografted 

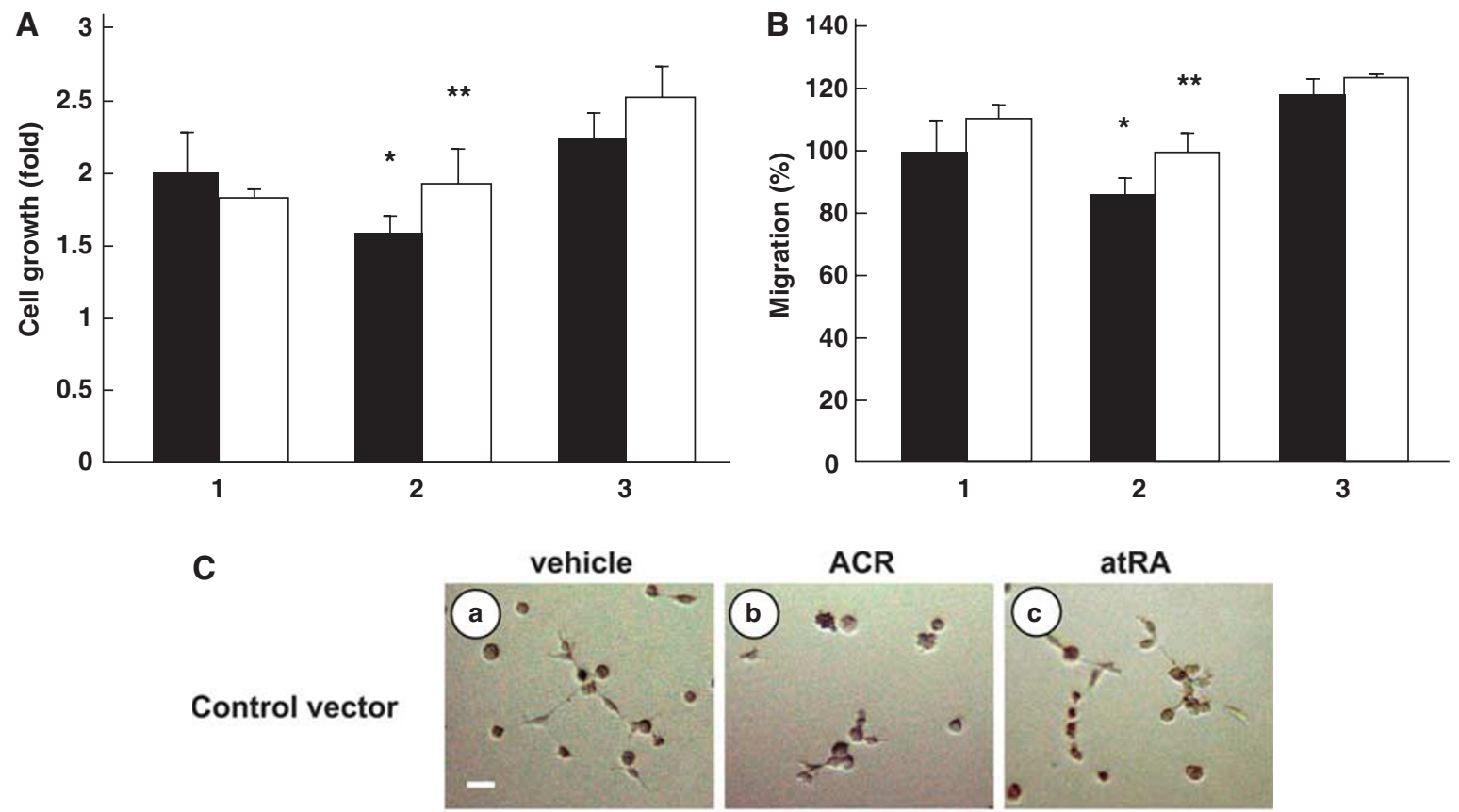

$(100 \pm 10)$

$(35 \pm 8)^{*}$

$(93 \pm 8)$

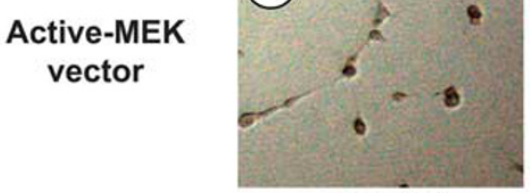

$(108 \pm 6)$

1

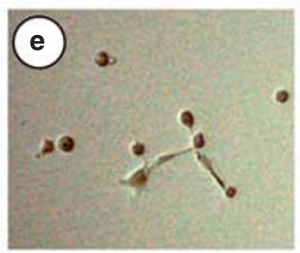

$(51 \pm 4) * *$

2

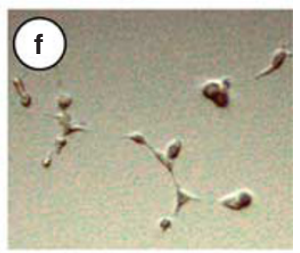

$(98 \pm 4)$

3

Figure 4 Effects of ACR and atRA on endothelial cell growth, migration, and tube formation. (A) HUVECs were transfected with a constitutively active MAPK kinase-expressing vector. After 2 days, cells $\left(1 \times 10^{5}\right.$ cells) were seeded onto $3.5-\mathrm{cm}$ dishes and incubated for another 2 days. They were then incubated for $48 \mathrm{~h}$ in $\alpha$-MEM medium containing $10 \%$ fetal calf serum, $100 \mathrm{ng} / \mathrm{ml} \mathrm{VEGF}$, and $5 \mu \mathrm{M} \mathrm{ACR}$ or atRA. Cells were counted and cell numbers are plotted as ploidy relative to values for untreated control cells at the start of incubation with ACR or atRA. Values represent means \pm s.e. $(n=2)$. Lane 1 , vehicle ( $0.1 \%$ ethanol); lane $2, A C R$; lane 3 , atRA. Closed columns, cells overexpressing control vector; open columns, cells overexpressing constitutively active MAPK kinase. A single asterisk indicates a significant difference $(P<0.05)$ from the control (lane 1 , closed column) and double asterisks indicate a significant difference $(P<0.05)$ between samples with or without the overexpression of constitutively active MAPK kinase.

(B) HUVECs were transfected with a constitutively active MAPK kinase-expressing vector. After 2 days, cells were wounded with a tip of pipette and incubated for $12 \mathrm{~h}$ in $\alpha$-MEM medium containing $2.5 \%$ fetal calf serum, $100 \mathrm{ng} / \mathrm{ml} \mathrm{VEGF}$, and $5 \mu \mathrm{M} \mathrm{ACR}$ or atRA. The numbers of cells that migrated into the denuded area were counted and are plotted as percentages relative to values for untreated control cells. Values represent means $\pm s$.e. $(n=2)$. Lane 1 , vehicle $(0.1 \%$ ethanol); lane $2, A C R$; lane 3 , atRA. Closed columns, cells overexpressing control vector; open columns, cells overexpressing constitutively active MAPK kinase. A single asterisk indicates a significant difference $(P<0.05)$ from the control (lane 1 , closed column) and double asterisks indicate a significant difference $(P<0.05)$ between samples without or with the overexpression of constitutively active MAPK kinase. (C) HUVECs were transfected with a constitutively active MAPK kinase-expressing vector. After 2 days, cells were seeded onto polymerized matrigel at $2 \times 10^{5}$ cells $/$ well. Thereafter, $100 \mathrm{ng} / \mathrm{ml}$ VEGF and $5 \mu \mathrm{M} \mathrm{ACR}$ or atRA were added, and incubated for $6 \mathrm{~h}$. Patterns of tube formation were photographed. Scale bar, $100 \mu \mathrm{m}$. Panels (a-c), cells overexpressing control vector; panels (d-f), cells overexpressing constitutively active MAPK kinase. Panels (a and $\mathbf{d}$ ), vehicle ( $0.1 \%$ ethanol) plus $100 \mathrm{ng} / \mathrm{ml} \mathrm{VEGF;} \mathrm{panels} \mathrm{(b} \mathrm{and} \mathbf{e}), 5 \mu \mathrm{M}$ ACR plus $100 \mathrm{ng} / \mathrm{ml}$ VEGF; panels (c and $\mathbf{f}), 5 \mu \mathrm{M}$ atRA plus $100 \mathrm{ng} / \mathrm{ml}$ VEGF. The numbers of branches in each micrograph were counted and the relative values are presented as percentages under each photograph. A single asterisk indicates a significant difference $(P<0.05)$ from the control (panel a vs panel b) and double asterisks indicate a significant difference $(P<0.05)$ between samples with or without the overexpression of constitutively active MAPK kinase (panel $\mathbf{b} v s$ panel $\mathbf{e}$ ). Panels A-C show representative results from two independent experiments, both of which gave similar results.

CAM model (Figure 7). Although $5 \mu \mathrm{M}$ ACR inhibited HCCinduced angiogenesis by $37 \%$ (panel b), the same concentration of atRA did not show any inhibition at all (panel c). This result suggested that ACR, but not atRA, may prevent the recurrence of HCC in part through the inhibition of cancer angiogenesis. 


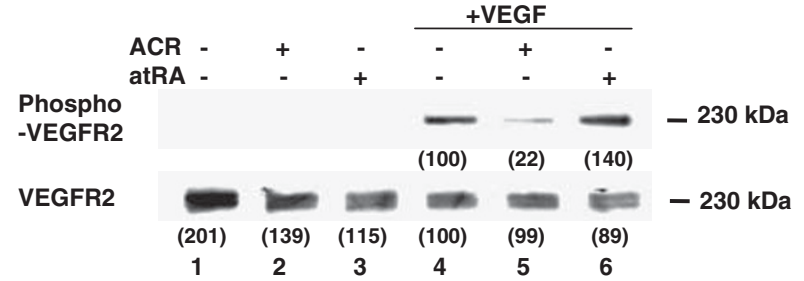

b

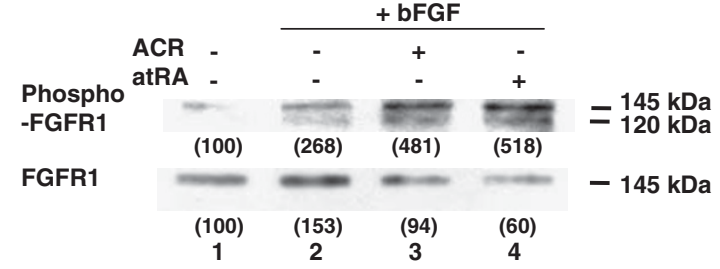

Figure 5 Effects of ACR and atRA on phosphorylation of growth factor receptors. After HUVECs had been incubated for $24 \mathrm{~h}$ with or without $5 \mu \mathrm{M}$ ACR or atRA in medium containing $2.5 \%$ serum, cells were stimulated with either $100 \mathrm{ng} / \mathrm{ml}$ VEGF (panel a) or $50 \mathrm{ng} / \mathrm{ml} \mathrm{bFGF} \mathrm{(panel} \mathrm{b)} \mathrm{for} 5 \mathrm{~min}$, and then lysed immediately. The amounts of each phosphorylated receptor (each upper bands), as well as whole amounts of each receptor (each lower bands), were assessed as described in the Materials and methods section. Panels $\mathbf{a}$ and $\mathbf{b}$ show representative results from two independent experiments, both of which gave similar results.

a

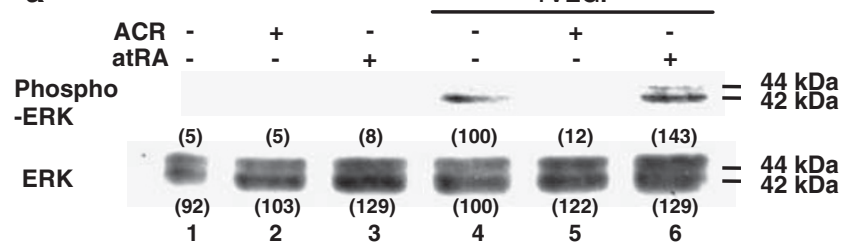

b

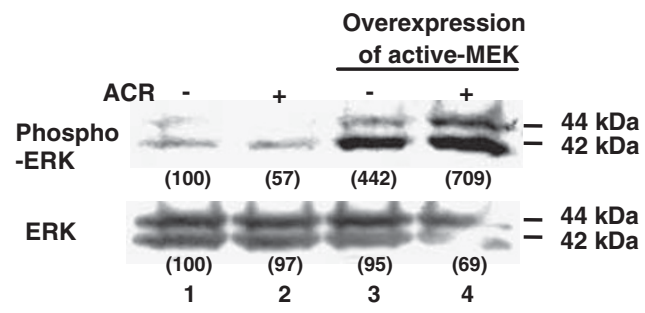

Figure 6 Effect of ACR on phosphorylation of ERK. (a) After HUVECs had been incubated for $24 \mathrm{~h}$ with or without $5 \mu \mathrm{M} \mathrm{ACR}$ or atRA in medium containing $2.5 \%$ serum, cells were stimulated with $100 \mathrm{ng} / \mathrm{ml}$ VEGF for $5 \mathrm{~min}$ and then lysed immediately. (b) HUVECs were transfected with a constitutively active $M E K$ gene. The day after transfection, the medium was changed and cells were treated with $5 \mu \mathrm{M} \mathrm{ACR}$ and lysed immediately. The amounts of each phosphorylated ERK (upper bands), as well as whole amounts of ERK (lower bands), were assessed as described in the Materials and methods section.

\section{DISCUSSION}

In this paper, we have shown an antiangiogenic activity of ACR and its underlying molecular mechanism, differing from that of atRA. Although the relative antiangiogenic activity of ACR was about 10 times weaker than that of atRA at the same concentrations (Figure 1), ACR showed much stronger inhibition in endothelial cell growth, migration, and tube formation than atRA (Figure 4) because of suppression in the VEGF-MAPK pathway (Figures 5 and 6). ACR suppressed the phosphorylation of VEGFR2 (Figure 5a, lane 5). On the other hand, atRA induced the phosphorylation of VEGFR2 (Figure 5a, lane 6). This might be because of the induction of VEGF by atRA as reported previously. ${ }^{17}$ ACR did not affect the levels of VEGF mRNA and the transactivation of the VEGF promoter (data not shown). ACR slightly inhibited the phosphorylation of VEGFR1 at $300 \mu \mathrm{M}$ but not at all at $30 \mu \mathrm{M}$ (Ishibashi et al, unpublished data). ACR (1 or $10 \mu \mathrm{M})$ did not inhibit other tyrosine kinases (for example, EGFR, FGFR3, FLT3, IGF1R, MET, PDGFR- $\alpha$, PDGFR- $\beta$, and TRKB) (Ishibashi et al, unpublished data). Moreover, ACR inhibited the phosphorylation of Ras but not the phosphorylation of Akt (Supplementary Figure 2). Therefore, we speculate that ACR may selectively interfere with the phosphorylation of VEGFR2 after Ras activation, although it is not clear how ACR does this; the underlying detailed molecular mechanism(s) remain to be elucidated. ACR and atRA enhanced the phosphorylation of FGFR1 (Figure 5b, lanes 3 and 4). This result might be because of the induction of bFGF by atRA as previously reported. ${ }^{18}$ ACR might also induce bFGF, probably through its retinoid activity.

These results suggest that suppression of the recurrence of HCC by ACR was induced in part through its antiangiogenic property by directly suppressing endothelial growth, migration, and tube formation through inhibition of the VEGFR2MAPK axis. On the other hand, atRA inhibits angiogenesis by a disruption of vascular networks through an increased expression of Ang2 and inhibition in the Ang/Tie2 pathway. ${ }^{6}$ Compared with atRA, ACR has 1/10-1/100 weaker 'retinoid' activities. $^{19}$ It has a $1 / 10$ weaker action on leukemia differentiation, ${ }^{20}$ and a $1 / 100$ weaker carcinogenic action in transgenic mice that express the dominant-negative form of retinoic acid receptor. ${ }^{21,22}$ On the other hand, accumulating evidence shows that ACR, but not atRA, has apoptosisinducing activity in HCC cells, as well as in smooth muscle cells and vascular neointima. ${ }^{23,24}$ We found that ACR acts as either a kinase inhibitor or a phosphatase stimulator and prevents hyperphosphorylation of RXR, ${ }^{9}$ and now VEGFR2. In this context, we speculate that ACR resembles geranylgeraniol in terms of its isoprenoid-like structure, which has been implicated in the modulation of many phosphorylation/dephosphorylation signaling. ${ }^{25,26}$

Hepatocellular carcinoma is a major cause of cancer mortality worldwide, especially in Southeast Asia and in subSaharan Africa. ${ }^{27}$ The development of HCC is frequently associated with a chronic inflammation of the liver induced by persistent infection with hepatitis B virus or hepatitis C virus. The annual incidence rises to $\sim 20-25 \%$ in cirrhotic patients who have undergone a potentially curative removal of primary HCC in Japan; the recurrence rate at 5 years after the curative treatment may exceed $70 \%$. This high recurrence rate is not because of local recurrence or metastasis from the original lesion, but rather from a second primary lesion. ${ }^{19}$ 

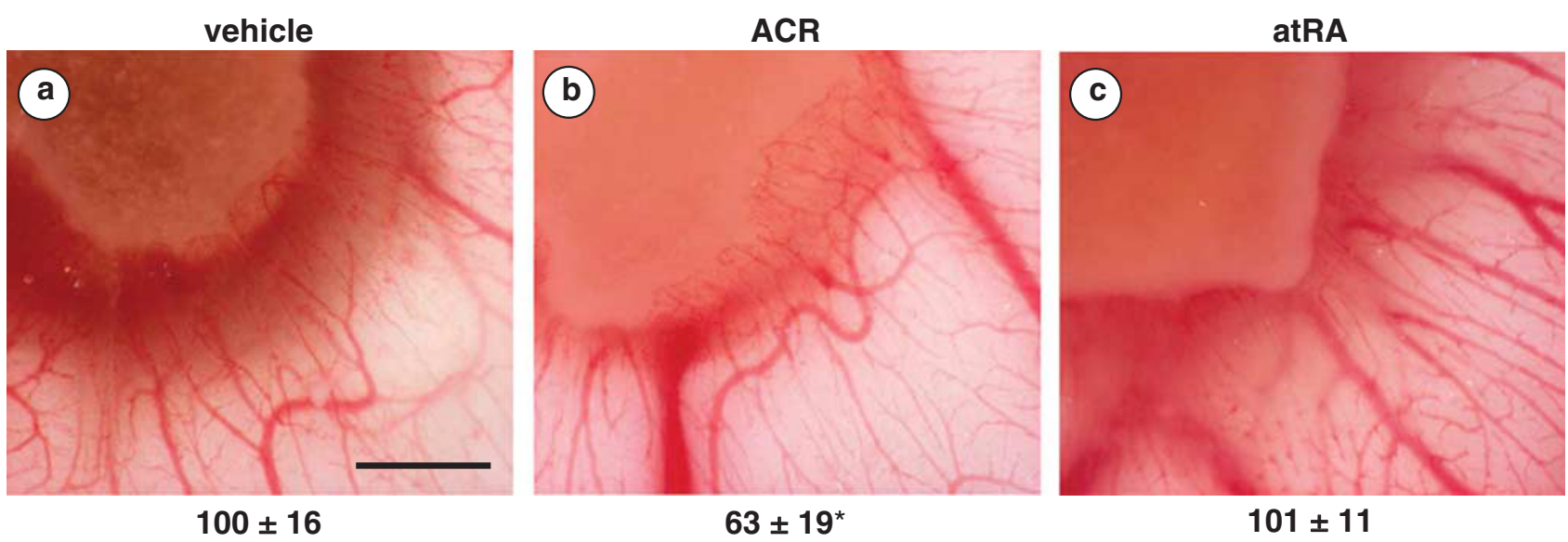

Figure 7 Effect of ACR on HCC-induced angiogenesis on CAM. HepG2 cell suspensions with or without $5 \mu \mathrm{M} \mathrm{ACR}$ or atRA were delivered at $4 \times 10^{5}$ cells per embryo onto the top of CAM on day 8 using a gelatin sponge implant. After a further 4-day incubation, a fat emulsion was injected into the chorioallantois, so that the vascular networks stood out against the white background of the lipid, and patterns of angiogenesis toward the implant were photographed. Panel (a), vehicle (1\% EtOH); panel (b), $5 \mu \mathrm{M}$ ACR; panel (c), $5 \mu \mathrm{M}$ atRA. Scale bar, $1 \mathrm{~mm}$. Total numbers of branches of blood vessels were counted and the relative values are presented as percentages under each photograph. An asterisk indicates a significant difference $(P<0.05)$ from the control. This result shows the representative result from two independent experiments, both of which gave similar results.

In HCC tissues, RXR- $\alpha$ is constitutively phosphorylated by the activation of MAPK, resulting in a loss of its function and accumulation of inactive RXR- $\alpha$ s inside cells as dominantnegative inhibitors. ${ }^{9,10}$ Therefore, phosphorylation of RXR- $\alpha$ causes a reduction in transactivation through a RAR/RXR complex. ${ }^{9,28}$ ACR inhibits the phosphorylation of RXR- $\alpha$ and restores the function of RXR- $\alpha$, and thereby transactivation through the RAR/RXR complex with endogenous retinoic acid. Retinoids are thought to activate the transcription of cell cycle inhibitor $\mathrm{p}_{2} 1^{\mathrm{CIP} 1}$ by $\mathrm{RAR}^{29}$ and by apoptosisinducer tissue transglutaminase in HCC. ${ }^{19}$ However, $5 \mu \mathrm{M}$ ACR hardly induces endothelial cell death (Figure 4A) and expression of $p 21^{C I P 1}$ mRNA levels in endothelial cells (data not shown). We found that phosphorylation of RAR/RXR was associated with the growth of endothelial cells and that ACR inhibited this phosphorylation (Supplementary Figure 3). As this phosphorylation of RAR/RXR coincided with the activation of VEGFR2 and Ras and as we had already found that RAR/RXR phosphorylation was induced by the overexpression of active MEK in cancer cells, ${ }^{10}$ we speculate that phosphorylation of RAR/RXR would occur downstream of the VEGF/VEGFR axis in growing endothelial cells.

Acyclic retinoid suppressed both angiogenesis and HCCinduced angiogenesis in a xenografted CAM model (Figure 1, panels b-d and Figure 7, panel b, respectively). In this model, ACR did not affect the preexisting blood vessels on CAM. These results suggest that ACR did not affect the mature blood vessel and only affected neovasculature formation, including both normal angiogenesis and tumor angiogenesis. On the other hand, atRA suppressed angiogenesis but failed to suppress the tumor angiogenesis on CAM (Figures 1 and 7, panels e-g and panel c, respectively), suggesting that atRA inhibits angiogenesis in the embryonic stage, but not tumor- induced angiogenesis, and addressing the superiority of ACR as a promising antitumor angiogenesis agent. The dose of ACR used in this experiment $(5 \mu \mathrm{M})$ is higher than the maximal blood concentration of ACR in clinical use $(1 \mu \mathrm{M})$ (Ishibashi et al, unpublished data). However, we believe that liver tissue has higher doses of ACR than does the blood level because of the accumulation of ACR in the liver. These results suggest that one of the mechanisms of ACR action to prevent recurrence of HCC is its antiangiogenic activity.

Supplementary Information accompanies the paper on the Laboratory Investigation website (http://www.laboratoryinvestigation.org)

\section{ACKNOWLEDGEMENTS}

The authors thank Dr NG Ahn (University of Colorado, CO) for providing the constitutively active MAPK kinase vector. This study was supported in part by grants from the Chemical Genomics Research Project from RIKEN (to SK) and Grant-in-Aids from the Ministry of Education, Science, Sports, and Culture (17015016, HM).

\section{DISCLOSURE/CONFLICT OF INTEREST}

The authors declare no conflict of interest.

1. Mazitschek R, Giannis A. Inhibitors of angiogenesis and cancer-related receptor tyrosine kinase. Curr Opin Chem Biol 2004;8:432-441.

2. Ferrara N, Kerbel R. Angiogenesis as a therapeutic target. Nature 2005;438:967-974.

3. Chambon P. A decade of molecular biology of retinoic acid receptors. FASEB J 1996;10:940-954.

4. Obora A, Shiratori $Y$, Okuno $M$, et al. Synergistic induction of apoptosis by acyclic retinoid and interferon- $\beta$ in human hepatocellular carcinoma cells. Hepatology 2002;36:1115-1124.

5. Altucci L, Leibowitz MD, Ogilvie KM, et al. RAR and RXR modulation in cancer and metabolic disease. Nat Rev Drug Discov 2007;6:793-810.

6. Suzuki $Y$, Komi $Y$, Ashino $H$, et al. Retinoic acid controls blood vessel formation by modulating endothelial and mural cell interaction via suppression of Tie2 signaling in vascular progenitor cells. Blood 2004;104:166-169. 
7. Altucci L, Gronemever $\mathrm{H}$. The promise of retinoids to fight against cancer. Nat Rev Cancer 2001;1:181-193.

8. Muto $\mathrm{Y}$, Moriwaki $\mathrm{H}$, Ninomiya $\mathrm{M}$, et al. Prevention of second primary tumors by an acyclic retinoid, polyprenoic acid, in patients with hepatocellular carcinoma. N Engl J Med 1996;334: 1561-1568.

9. Matsushima-Nishiwaki $\mathrm{R}$, Okuno $\mathrm{M}$, Takano $\mathrm{Y}$, et al. Molecular mechanism for growth suppression of human hepatocellular carcinoma cells by acyclic retinoid. Carcinogenesis 2003;24: 1353-1359.

10. Matsushima-Nishiwaki R, Okuno M, Adachi $S$, et al. Phosphorylation of retinoid $X$ receptor $\alpha$ at serine 260 impairs its metabolism and function in human hepatocellular carcinoma. Cancer Res $2001 ; 61$ : 7675-7682.

11. Kagawa M, Tetsuo S, Ishibashi N, et al. An acyclic retinoid, NIK-333, inhibits $\mathrm{N}$-diethylnitrosamine-induced rat hepatocarcinogenesis through suppression of TGF- $\alpha$ expression and cell proliferation. Carcinogenesis 2004;25:979-985.

12. Komi $Y$, Suzuki $Y$, Shimamura $M$, et al. Mechanism of inhibition of tumor angiogenesis by $\beta$-hydroxyisovalerylshikonin. Cancer Sci 2009;100:269-277.

13. Mansour SJ, Matten WT, Hermann AS, et al. Transformation of mammalian cells by constitutively active MAP kinase kinase. Science 1994;265:966-970.

14. Komi Y, Ohno O, Suzuki Y, et al. Inhibition of tumor angiogenesis by targeting endothelial surface ATP synthase with sangivamycin. Jpn J Clin Oncol 2007;37:867-873.

15. Rabatti D, Nico B, Vacca A, Presta M. The gelatin spongechorioallantoic membrane assay. Nat Protoc 2006;1:85-91.

16. Chen MJ, Chiou PP, Lin P, et al. Suppression of growth and cancerinduced angiogenesis of aggressive human breast cancer cells (MDA-MB-231) on the chorioallantoic membrane of developing chicken embryos by E-peptide of pro-IGF-I. J Cell Biochem 2007;101:1316-1327.

17. Saito A, Sugawara A, Uruno A, et al. All-trans retinoic acid induces in vitro angiogenesis via retinoic acid receptor: possible involvement of paracrine effects of endogenous vascular endothelial growth factor signaling. Endocrinology 2007;148:1412-1423.
18. Gaetano C, Catalano A, Illi B, et al. Retinoids induce fibroblast growth factor-2 production in endothelial cells via retinoic acid receptor $\alpha$ activation and stimulate angiogenesis in vitro and in vivo. Circ Res 2001;88:E38-E47.

19. Kojima S, Okuno M, Matsushima-Nishiwaki R, et al. Acyclic retinoid in the chemoprevention of hepatocellular carcinoma (Review). Int J Oncol 2004;24:797-805.

20. Tsurumi H, Tojo A, Takahashi $\mathrm{T}$, et al. Differentiation induction of human promyelocytic leukemia cells by acyclic retinoid (polyprenoic acid). Int J Hematol 1993;59:9-15.

21. Sakabe $T$, Tsuchiya $H$, Endo $M$, et al. An antioxidant effect by acyclic retinoid suppresses liver tumor in mice. Biochem Pharmacol 2007;73:1405-1411.

22. Yanagitani A, Yamada S, Yasui $S$, et al. Retinoic acid receptor $\alpha$ dominant negative form causes steatohepatitis and liver tumors in transgenic mice. Hepatology 2004:40:366-375.

23. Nakamura N, Shidoji Y, Yamada Y, et al. Induction of apoptosis by acyclic retinoid in the human hepatoma-derived cell line, HuH-7. Biochem Biophys Res Commun 1995;207:382-388.

24. Kada N, Suzuki T, Aizawa K, et al. Acyclic retinoid inhibits neointima formation through retinoic acid receptor beta-induced apoptosis. Arterioscler Thromb Vasc Biol 2007;27:1535-1541.

25. Nakajo S, Okamoto M, Masuda Y, et al. Geranylgeraniol causes a decrease in levels of calreticulin and tyrosine phosphorylation of a 36-kDa protein prior to the appearance of apoptotic features in HL-60 cells. Biochem Biophys Res Commun 1996;226:741-745.

26. Hashimoto K, Morishige K, Sawada K, et al. Geranylgeranylacetone inhibits lysophosphatidic acid-induced invasion of human ovarian carcinoma cells in vitro. Cancer 2005;103:1529-1536.

27. Ince N, Wands JR. The increasing incidence of hepatocellular carcinoma. N Engl J Med 1999;340:798-799.

28. Solomon C, White $\mathrm{JH}$, Kremer R. Mitogen-activated protein kinase inhibits 1,25-dihydroxyvitamin D3-dependent signal transduction by phosphorylating human retinoid $X$ receptor $\alpha$. J Clin Invest 1999;103:1729-1735.

29. Liu M, lavarone A, Freedman LP. Transcriptional activation of the human p21(WAF1/CIP1) gene by retinoic acid receptor. J Biol Chem 1996;271:31723-31728. 